\title{
Transanal Endorectal Pullthrough for Hirschsprung's Disease Without Frozen-Section Biopsy Facility
}

\author{
CHOUDHURY KM ${ }^{\mathrm{a}}$, JAFOR MA ${ }^{\mathrm{b}}$, AHMED SU $^{\mathrm{c}}$
}

\begin{abstract}
:
Objective: The purpose of this study was to evaluate results of treatment of patients with Hirschsprung's disease (HPD) by transanal endorectal pull-through (TEPT) in a setting where frozen-section biopsy facility was not available.

Methods: From November 2001 to September 2007, fortytwo patients with HPD had undergone TEPT in Rajshahi Medical College Hospital (RMCH) and at various clinics in Rajshahi. The age of the patients ranged from 7 days to 12 years. In all cases, the extent of aganglionosis was limited to recto-sigmoid region and was assessed by the three classical features of contrast enema namely spastic aganglionic zone, transition zone (TZ) and proximal dilated zone (PDZ). During operative pull-through, transition zone (TZ) and proximal dilated zone (PDZ) were identified by naked eye and correlated with $x$-ray findings. Eleven patients had colostomy fashioned prior to the TEPT procedure. Followup was done for 14 to 58 months (mean 36 months).
\end{abstract}

Result: Thirty-nine patients had satisfactory outcome without any complication. The mean operative time was 100 minutes. The average length of resected gut was $22 \mathrm{~cm}$. Post-operative recovery was smooth and oral feeding was resumed after a mean period of 48 hours. Of the eleven children with

Introduction:

Surgical treatment of Hirschsprung's disease (HPD) is undergoing dramatic changes with recent emphasis on minimal invasive procedure. Georgeson et $\mathrm{al}^{1}$ in 1995 first used transanal mucosectomy in 12 children. ${ }^{1}$ In 1998, De la Torre-Mondragon and Ortega-Salgado performed mucosectomy and pull-through by transanal

a. Dr. Kamal M. Choudhury, MS (pediatric surgery), Associate professor, Dept of Pediatric Surgery, BIRDEM General Hospital \& Ibrahim Medical College.

b. Dr. Md. Abu Jafor, MS (pediatric surgery), Professor (cc), Dept of Pediatric Surgery, Sir Salmullah Medical College \& Mitford Hospital.

c. Dr. Safiruddin Ahmed, FCPS (surgery), Ex-Professor (cc), Dept of Pediatric Surgery, Rajshahi Medical College Hospital.

Address of correspondence: Dr. Kamal M. Choudhury, Associate Professor, Dept of pediatric surgery, BIRDEM. Email:kamalemcee@gmail.com

Received : 13 July, 2013

Accepted : 10 February 2014 colostomy, 6 were older than 10 years with huge megacolon, and the remaining five had their colostomy done at the referring hospital. In 4 patients out of 42 , naked eye assessment of the TZ and PDZ and their correlation with the $x$-ray features were not possible. In such situation, a few $\mathrm{cm}$ more of the proximal ganglionic gut from $P D Z$ was resected. Three children out of 42 developed complications; postoperative enterocolitis in two and intra-abdominal bleeding from sigmoid mesocolon in one. There was no incidence of cuff abscess, anastomotic leak, retained segment, encopresis or constipation.

Conclusion: The minimal invasive procedure TEPT involves less time with trifle morbidity and offers rapid recovery with shorter hospital stay. The functional outcome is also excellent with small complication rate. The naked eye correlation of the morbid features of rectum and colon in HPD with classical $x$-ray features is reasonably consistent and TEPT can safely be performed where frozen-section facility is not available.

Key Words: Hirschsprung's disease, Transanal endorectal pull-through, Frozen-section biopsy.

(Birdem Med J 2014; 4(2): 84-87)

approach without laparoscopy. ${ }^{2}$ Their experience involved 5 patients with a follow-up ranging 6 to 15 months. Langer et al and Albanese et al also reported similar experience of transanal endorectal pull-through (TEPT). ${ }^{3,4}$ Here we describe our experience of TEPT in a setting where frozen-section biopsy facility is not available.

Materials \& Methods:

From November 2001 to September 2007, forty-two patients had undergone TEPT in RMCH and at different private clinics in Rajshahi. Their age ranged from 7 days to 12 years. Diagnosis in all cases was made with combination of contrast enema and rectal biopsy. The five neonates with colostomy performed at the referring hospital on the basis of contrast enema had later undergone rectal biopsy for confirmation of the diagnosis. In each patient, the extent of aganglionosis was determined by the typical radiological features 
namely the spastic aganglionic zone, transition zone (TZ) and proximal dilated zone (PDZ) seen in contrast enema. The patients with aganglionosis extending beyond recto-sigmoid region were excluded from the study. Also the patients with equivocal radiological finding of contrast enema in respect to the level of aganglionosis were excluded from the study. By radiological evaluation, 17 patients had rectal aganglionosis and remaining 25 had recto-sigmoid aganglionosis. These were consistent with the histopathological reports obtained post-operatively.

During operative pull-through, TZ and PDZ were distinctly discerned in 38 patients. The remaining 4 patients with unremarkable naked-eye feature of the rectum and colon were all neonates with colostomy fashioned at an early age. In such situations, where TZ was indistinct, resection was done at a level $5-10 \mathrm{~cm}$ proximally in the PDZ to circumvent the probability of retaining any affected segment.

Detailed counseling with the parents of the patients was done regarding the unavailability of the frozensection facility and the potential risk of complication that may result, and written consent was taken from each party.

Outcome was evaluated with respect to operative time, pain-relief measures, time before resumption of oral feed, hospital stay, incidence of enterocolitis, constipation and encopresis.

The standard principles of TEPT was followed regarding pre-operative preparations of the patients and for operative procedures. ${ }^{2-4}$ Mucosal dissection was begun $0.5 \mathrm{~cm}$ above the dentate line and was extended 5 to 8 $\mathrm{cm}$ proximally. Peri-rectal space was then entered and full-thickness dissection of the rectum and colon undertaken. The evaluation of the morbid anatomy of the rectum and colon was then done and resection of the large gut carried out. The muscle cuff was divided posteriorly. The colo-anal anastomosis was performed with interrupted absorbable stitches.

A few measures were adopted which were found helpful at surgery and also appear beneficial for the patients. For example: (1) to use a Foley's catheter, (2) to put sutures on the intervening positions in between 12,3, 6 and 9 o'clock marks, (3) to start sub-mucosal dissection at 3 o'clock and end at 9 o'clock position, (4) to split muscle cuff posteriorly, (5) to make a mucosal sheath of at least $5 \mathrm{~cm}$ in length, (6) to taper the proximal colon when markedly dilated, (7) meticulous colo-anal stitches to prevent stricture formation.

Results:

The study consisted of 11 neonates, 13 infants, 10 children between 1 and 5 years and 8 children older than 5 years. Of the 42 patients, 29 were boys (M: $\mathrm{F}=$ 2.2:1.0). About $60 \%$ patients had recto-sigmoid HPD ( 25 out of 42) and the remaining 17 had rectal HPD. The characteristics of the patients diagnosed as HPD was shown in Table 1.

\section{Table-I}

Characteristics of patients with HPD

\begin{tabular}{lc} 
& TEPT $(\mathrm{n}=42)$ \\
\hline Age: $<1$ month & $11(26.2 \%)$ \\
$\quad: 1$ month- 1 year & $13(30.9 \%)$ \\
$\quad: 1$ year -5 years & $10(23.8 \%)$ \\
$\quad:>5$ years & $08(19.1 \%)$ \\
Male: Female & $2.2: 1.0$ \\
Age at TEPT (month) & $38.1(0.33-144)$ \\
Weight at TEPT $(\mathrm{kg})$ & $14.6(2.6-27)$ \\
Transition zone (rectal) & 17 \\
Transition zone (recto-sigmoid) & 25 \\
\hline
\end{tabular}

The mean age and weight of the patients at the time of operative pull-through were 38.1 months and $14.6 \mathrm{~kg}$ respectively. The operative time ranged from 60 minutes to 150 minutes (mean 100 minutes). The variation was due to various factors viz. age of the patients (with younger patients requiring less operating time), adherent mucosa, previous enterocolitis (leads to mucosal adherence), intra-operative bleeding ( more likely in older children). The length of the resected gut varied from $12 \mathrm{~cm}$ to $35 \mathrm{~cm}$ (mean $22 \mathrm{~cm}$ ). The outcome measures are shown in Table-II.

Thirty-nine children appeared to have excellent result and did not have any complication. The mean duration for which post-operative analgesia was required was 1.5 days. Younger children had only acetaminophen for relief of pain while diclofenac sodium was used for older children. Oral feed was started 1 to 4 days (mean 2 days) post-operatively. The mean hospital stay was 3.5 days (2 - 10 days). 


\section{Table-II}

\section{Outcome measures}

\begin{tabular}{lc}
\hline Operating time (minutes) & $100(60-150)$ \\
Length of resected gut $(\mathrm{cm})$ & $22(12-35)$ \\
Pain-relief requirement (day) & $1.5(1-5)$ \\
Resumption of oral feed (day) & $2(1-4)$ \\
Post-operative hospital stay (day) & $3.5(2-10)$ \\
Post-operative enterocolitis (no. of patient) & $2(4.76 \%)$ \\
Secondary surgery (no. of patient) & $1(2.38 \%)$ \\
\hline
\end{tabular}

The complication rate was $7.1 \%$ in this study. Two patients developed enterocolitis 3 months and 8 months post-operatively and both required hospitalization for providing bowel rest and intravenous antibiotics. ${ }^{5}$ Another patient developed intra-operative bleeding while dissecting the sigmoid mesocolon for sigmoid mobilization. The active bleeding from the proximal end of the vascular mesocolon was then controlled by laparotomy. This was the only patient who needed blood transfusion. None of the patient in the series had constipation or encopresis as complication.

\section{Discussion:}

It is now widely recognized that TEPT may provide several advantages such as shorter operating time, rapid and less morbid convalescence, less hospital stay with commendable surgical outcome ${ }^{2-4}$. The results also show lesser complication rate particularly those related to injury to pelvic nerves ${ }^{4}$. Our results also showed similar satisfactory outcome in spite of the fact that frozen-section facility was not available. The mean operating time was 100 minute which conforms to most series $^{6}$. The mean age at TEPT was 38.1 months. Comparison with other literatures showed a higher mean for age at TEPT in our series. ${ }^{7}$ This may be due to poor socio-economic condition where delayed treatmentseeking is common and inappropriate referral is usual. The other outcome parameters namely pain-relief requirement, resumption to oral feed, and hospital stay were all consistent with various experiences. ${ }^{3,8}$

The overall complication rate was $7.1 \%$ which was less than what reported in few series, ${ }^{4,9}$ but was similar to some study $1,2,10$. Two children suffered from enterocolitis 3 months and 8 months post-operatively and were improved after treatment. The other child developed intra-operative bleeding from vessels in the sigmoid mesocolon for which laparotomy was done to control bleeding. A laparoscopic approach (which was not available at the center) would have avoided the necessity of laparotomy. To prevent such complication one has to be very cautious and meticulous during mobilization of the sigmoid colon.

Though TEPT is usually performed without a prior colostomy, eleven of our patients had colostomies. Five of them (all neonates) had their colostomies fashioned elsewhere. The other six were older than 10 years and they had hugely dilated colon with numerous large and hard scybullae palpable abdominally. Repeated rectal wash-out did not effectively evacuate of the large gut. Fashioning a colostomy in those circumstances was deemed an appropriate option. An alternative of colostomy could have been to taper the dilated colon during the pull-through procedure. One should keep in mind that tapering would increase the possibility of contamination and lengthen the operating time with likelihood of increased post-operative complications and morbidity.

In this study, we did not use state-of-the-art service of frozen-section biopsy facility (because of unavailability). Rather, we relied on the contrast enema x-rays for the assessment of the extension of aganglionosis in each patient. Classic radiological features viz. the spastic aganglionic zone, the transition zone (TZ) and the proximal dilated zone (PDZ) were the basis for a radiological diagnosis and evaluation of $\mathrm{HPD}^{11-15}$. Proctor ML et al ${ }^{16}$ showed that in $90 \%$ instances the pathologic and radiographic TZs were concordant in rectosigmoid HPD. In our series, the concordance rate was $90.5 \%$ (38 patients in 42). That is, in 4 discordant patients who were all neonates, the pathological TZs were not distinct. These neonates had their colostomy fashioned elsewhere at an early age ${ }^{17}$. However, the pathological TZs were evident in other seven patients having colostomy. Six out of these 7 patients were older than 10 years. This reflects that fashioning colostomy early may obliterate the distinct transition zones (TZs) in HPD.

Question may be raised whether there is increased chance of potential risks of complications if TEPT is done without the aid of frozen-section biopsy facility; particularly the potential risk of leaving a retained aganglionic segment after colo-anal anastomosis. This 
can be minimized if the pathologic TZ (when not distinctly evident during surgery) is mentally extrapolated from the radiographic $\mathrm{TZ}$ and then resection is done at a level $5-10 \mathrm{~cm}$ more proximally ${ }^{18}$. This was also practiced when retained hard stool was stuck in the proximal dilated zone (PDZ) as, in such condition, the radiographic $\mathrm{TZ}$ would appear more distal ${ }^{12}$.

From this study, we can surmise that in 38 cases out of 42 (i.e. $90.5 \%$ ), the radiographic TZs did correspond to the pathologic TZs at surgery. Only in 4 cases $(9.5 \%)$, the pathological TZs were not evident in the morbid colon at the time of surgery. In no case, the radiographic TZ mismatched with the pathologic TZ. We attribute the cause of absence or obliteration of pathologic TZ in 4 cases to fashioning of colostomy at an early neonatal age. So, if we perform TEPT without colostomy the likelihood of the TZ being obscured would be less likely and it may be justifiable to perform the minimal invasive procedure even without frozen-section biopsy facility (if unavailable). Because, studies had found that in rectosigmoid HPD with evident radiographic TZs, 90\% patients had a matching level of aganglionosis. ${ }^{16}$ In the same study, it was also found that discordance is common in long-segment and total colonic HPD. Moreover, Rosenfield found the radiographic TZs matched the level of aganglionosis identified at the time of surgery in all 27 patients. ${ }^{14}$ From these, it can be concluded that where frozen-section facility is unavailable, TEPT can be performed safely with occurrence of complication rate within acceptable range, and while the small incidence of discordance between anticipated level of aganglionosis and operative findings is borne in mind as a cautionary caveat.

\section{References:}

1. Georgeson KE, Fuenfer MM, Hardin WD. Primary laparoscopic pull-through for Hirschsprung's disease in infants and children. J Pediatr Surg 1995;30:1017-22.

2. Del Torre-Mondragon L, Ortega-Salgado JA. Transanal endorectal pull-through for Hirschsprung's disease. J Pediatr Surg 1998; 33:1283-86.

3. Langer JC, Minkes RK, Mazzioti MV, et al. Transanal one-stage Soave [rocedure for infants with Hirschsprung's disease. J Pediatr Surg 1999; 34: 148-52.

4. Albanese CT, Jennings RW, Smith B, et al. Perineal onestage pull-through for Hirschsprung's disease. J Pediatr Surg 1999; 34: 377-80.
5. Liu DC, Rodriguez J, Hill CB, et al: Transanal mucosectomy in the treatment of Hirschsprung's disease. J Pediatr Surg. 2000; 35, 235-38.

6. Gao Y, Li G, Zhang X, et al. Primary transanal rectosigmoidectomy of Hirschsprung's disease: Preliminary results in the initial 33 cases. J Pediatr Surg 2001; 36: 1816-18

7 Langer JC, Scifert M, Minkes RK. One-stage Soave pullthrough for Hirschsprung's disease: A comparison of the transanal vs open approaches. J Pediatr Surg 2000; 35 : 820-22.

8 Saltzman DA, Telander MJ, Brennom WS, et al. Transanal mucosectomy: A modification of the Soave procedure for Hirschsprung's disease. J Pediatr Surg 1996; 31: 1272 275.

9 Teitelbaum DH, Drongowski RA, Chamberlain JN, et al: Long-term stooling patterns in infants undergoing primary endorectal pull-through for Hirschsprung's disease. J Pediatr Surg 1997; 32: 1049-53.

10. Hackam DJ, Superina RA, Pearl RH. Single-stage repair of Hirschsprung's disease: A comparison of 109 patients over 5 years. J Pediatr Surg 1997; 32: 1028-32.

11. Berman CZ. Roentgenographic manifestations of congenital megacolon (Hirschsprung's disease) in early infancy. Pediatrics 1956; 18: 227-38.

12. Evans WA, Willis R: Hirschsprung's disease. The roentgen diagnosis in infants. AJR 1957; 78: 1024-48.

13. BerdonWE, Baker DH. The roentgenographic diagnosis of Hirschsprung's disease in infancy. AJR 1965; 93: 43246.

14. Rosenfield NS, Ablow RC, Markowitz RI, et al. Hirschsprung's disease: Accuracy of barium enema examination. Radiology 1984; 150: 393-400.

15. Taxman TL, Yulish BS, Rothstein FC. How useful is the barium enema in the diagnosis of infantile Hirschsprung's disease? AJDC 1986; 140:881-84.

16. Proctor ML, Traubici J, Langer JC, et al. Correlation between radiographic transition zone and level of aganglionosis in Hirschsprung's disease: Implications for surgical approach. J Pediatr Surg 2003; 38: 775-78.

17. Ehrenpreis T: Megacolon in the newborn. Clinical and roentogenological study with pecial regard to pathogenesis. Acta Pediatr 1945; 32: 358-70.

18. Shimotake T, Tomiyama H, Aoi S, et al. Discrepancy between macroscopic and microscopic transition zones in Hirschsprung's disease with reference to the type of RET/GDNF/SOX10 gene mutation. J Pediatr Surg 2003; 38: 698-701. 